\title{
Nuevos registros y lista actualizada de las algas verdes (Chlorophyta) del litoral de Veracruz, México
}

\section{New records and updated list of green algae (Chlorophyta) from the littoral of Veracruz, Mexico}

\author{
Deisy Y. García-López*, luz Elena Mateo-Cid \& A. Catalina Mendoza-González \\ Instituto Politécnico Nacional, Escuela Nacional de Ciencias Biológicas, Departamento de Botánica, Carpio y Plan de Ayala, \\ Colonia Santo Tomás, 11340 Ciudad de México, México. \\ *deisyyazmin@gmail.com
}

\begin{abstract}
RESUMEN
Se llevó a cabo una revisión bibliográfica y de herbario de las algas verdes (Chlorophyta) que han sido registradas para el litoral de Veracruz, México. Además se incorporaron los datos obtenidos de muestreos realizados en doce localidades de 2006 a 2016. Se determinó la presencia de 93 especies de algas verdes, siendo la familia Cladophoraceae la mejor representada con 28 especies, seguida de Ulvaceae con 15 y Caulerpaceae con 12. La lista florística se acompaña de datos sobre estacionalidad, reproducción, hábitat y referencias. De los 93 taxa, 19 son nuevos registros para el litoral de Veracruz, y se cita por primera vez a Ulva profunda, Chaetomorpha vieillardii y Stigeoclonium tenue para la costa occidental del Atlántico de México. Por otro lado, se compara la riqueza específica entre las épocas climáticas de lluvias de invierno (nortes), secas y lluvias de verano durante el periodo de estudio. La riqueza específica más alta fue encontrada durante la época de lluvias de verano.
\end{abstract}

Palabras clave: Ulvophyceae, inventario, épocas climáticas, riqueza específica.

\begin{abstract}
The green algae (Chlorophyta) recorded in the literature and reported at herbaria for the littoral of Veracruz were reviewed, and data obtained from sampling during 2006 to 2016 were added for a total of 93 taxa. The Cladophoraceae family was the best represented with 28 taxa, followed by Ulvaceae with 15 and Caulerpaceae with 12 . The floristic list is accompanied by data on seasonality, reproduction, habitat and references. Of the 93 taxa, 19 are new records for littoral of Veracruz, with Ulva profunda, Chaetomorpha vieillardii and Stigeoclonium tenue being new records for the occidental coast of the Atlantic of Mexico. Species diversity was compared between the winter rains, dry and rainy seasons during the period study. The greatest diversity was found during the rainy season.
\end{abstract}

KEYWORDS: Ulvophyceae, inventory, seasonality, species richness.

\section{INTRODUCCIÓN}

Las algas verdes (Chlorophyta) son un importante componente del ecosistema marino del estado de Veracruz, ya que son productores primarios y formadoras de arena. A pesar de la extensión del litoral veracruzano, resalta lo reducido de las investigaciones sobre las algas marinas bentónicas de la costa de Veracruz (Ortega et al. 2001, Garduño Solórzano et al. 2005). Los primeros registros de algas marinas de la costa de Veracruz se encuentran en el trabajo de Liebmann (1846), posteriormente estos registros se enriquecieron con los estudios de Huerta-Múzquiz (1960), Humm \& Hildebrand (1962), De la Campa-De Guzmán
(1965) seguidos por los de Sánchez-Rodríguez (1967, 1980), Ramírez-Rodríguez (1975), Valenzuela (1987), Flores-Davis (1993) y Ramírez-Rodríguez et al. (2011). En estos trabajos se encuentran registradas 72 especies de algas verdes para el litoral de Veracruz. El objetivo del presente estudio es dar a conocer una lista florística de ocho localidades poco estudiadas de esta región, además de las escolleras de Tuxpan, Veracruz-Playa Penacho del Indio (Boca del Río) y las escolleras de Coatzacoalcos, aportando en ella nuevos registros de Chlorophyta para Veracruz, así como datos acerca de su distribución, reproducción y variación temporal durante las épocas climáticas de lluvias de invierno (nortes), secas y lluvias de verano. 


\section{MATERIALES Y MÉTODO}

ÁrEA DE ESTUDio

El litoral de Veracruz pertenece a la primera y segunda unidad morfotectónica del Golfo de México y Mar Caribe. La unidad I se extiende desde el Río Bravo, Tamaulipas, hasta Punta Delgada, Veracruz, con una longitud de $\sim 700$ $\mathrm{km}$. Se ubica dentro de la Planicie Costera Nororiental y limitada al sur con la Cordillera Neovolcánica. De norte a sur se tienen las siguientes subprovincias fisiográficas: Cuenca del Bravo, Cuenca del Río Purificación, Cuenca Pánuco-Tuxpan (Tampico-Nautla) y la porción NW de la Zona de Veracruz. Como rasgo mayor, también se considera la existencia de la Barra de Tuxpan, que es una isla de barrera localizada entre Tampico y Tuxpan, probablemente controlada por topografía submarina y por procesos marinos construccionales. Por lo que respecta a la unidad II, ésta se extiende desde Punta Delgada, Veracruz, hasta las inmediaciones de Coatzacoalcos, Veracruz, con un desarrollo aproximado de $300 \mathrm{~km}$. Incluye al norte parte del extremo oriental de la Cordillera Neovolcánica y en sus porciones central y sur a la Planicie Costera de Sotavento (Carranza-Edwards et al. 1975). El clima del litoral de Veracruz se distribuye de norte a sur desde el semiseco muy cálido y cálido BS1(h')w(i') y BSO(h')w"(x') hasta el cálido subhúmedo Aw0(i’)(gw") y cálido húmedo (Am) (García 1983). En el Golfo de México la temperatura está determinada por el influjo de la Corriente del Lazo, que transporta aguas superficiales, tiene forma de herradura y fluye en el sentido de las agujas del reloj, transfiriendo aguas cálidas subtropicales del Mar Caribe a través del Estrecho de Yucatán hacia el Golfo de México.

La capa superficial del agua de mar (hasta los $200 \mathrm{~m}$ de profundidad) presenta temperaturas fluctuantes. En los meses de invierno (diciembre, enero, febrero) se registran valores de 15 a $25^{\circ} \mathrm{C}$, debido a los frentes polares y nortes. Durante el verano (julio, agosto y septiembre) la temperatura alcanza $28^{\circ} \mathrm{C}$. Tales variaciones se deben a la influencia de las corrientes de El Lazo y la del Caribe, ambas cálidas. En el litoral de Veracruz las mareas son diurnas. Las aguas típicas del Golfo de México tienen una salinidad de 36.5 p.s.u., producto de la mezcla de los giros anticiclónicos con la masa de agua subtropical subsuperficial, que tiene una salinidad de 36,6 p.s.u. a una profundidad de $70 \mathrm{~m}$ (De la Lanza-Espino \& Gómez Rojas 2004).

LOCALIDADES DE MUESTREO

En la Tabla 1 se indican los sitios de muestreo con coordenadas geográficas. La ubicación se muestra en la Figura 1. Las características de cada localidad son las siguientes:

1. Escolleras de Tuxpan: escolleras formadas por grandes rocas, la localidad se encuentra influenciada por actividades de turismo.

2. Barra de Cazones: playa con pendiente suave y oleaje moderado a intenso, posee varias zonas rocosas expuestas, siendo en su mayoría arenosa con praderas de pastos marinos.

3. Lechuguillas: playa en su mayoría arenosa con numerosos cantos rodados.

4. Playa Los Muñecos: playa con acantilados y fuerte oleaje, además de zonas rocosas expuestas en donde se forman pozas intermareales.

TABLA 1. Localidades de estudio con localización geográfica. / Locations of study with geographic location.

\begin{tabular}{|c|c|c|c|}
\hline & LOCALIDAD & LATITUD N & LONGITUD O \\
\hline 1 & Escolleras de Tuxpan & $20^{\circ} 58^{\prime} 22^{\prime \prime}$ & $97^{\circ} 18^{\prime} 17^{\prime \prime}$ \\
\hline 2 & Barra de Cazones & $20^{\circ} 44^{\prime} 08^{\prime \prime}$ & $97^{\circ} 11^{\prime} 41^{\prime \prime}$ \\
\hline 3 & Lechuguillas & $20^{\circ} 00^{\prime} 22^{\prime \prime}$ & $96^{\circ} 34^{\prime} 36^{\prime \prime}$ \\
\hline 4 & Playa Los Muñecos & $19^{\circ} 44^{\prime} 38^{\prime \prime}$ & $96^{\circ} 24^{\prime} 27^{\prime \prime}$ \\
\hline 5 & Villa Rica & $19^{\circ} 40^{\prime} 14^{\prime \prime}$ & $96^{\circ} 23^{\prime} 37^{\prime \prime}$ \\
\hline 6 & Playa Paraíso & $19^{\circ} 35^{\prime} 14^{\prime \prime}$ & $96^{\circ} 23^{\prime} 05^{\prime \prime}$ \\
\hline 7 & Veracruz-Penacho del Indio & $19^{\circ} 09^{\prime} 04^{\prime \prime}$ & $96^{\circ} 05^{\prime} 34^{\prime \prime}$ \\
\hline 8 & Escolleras de Alvarado & $18^{\circ} 47^{\prime} 27^{\prime \prime}$ & $95^{\circ} 44^{\prime} 34^{\prime \prime}$ \\
\hline 9 & Punta Puntilla & $18^{\circ} 43^{\prime} 00^{\prime \prime}$ & $95^{\circ} 17^{\prime} 16^{\prime \prime}$ \\
\hline 10 & Playa Hermosa & $18^{\circ} 39^{\prime} 54^{\prime \prime}$ & $95^{\circ} 07^{\prime} 43^{\prime \prime}$ \\
\hline 11 & Monte Pío & $18^{\circ} 38^{\prime} 44^{\prime \prime}$ & $95^{\circ} 05^{\prime} 43^{\prime \prime}$ \\
\hline 12 & Escolleras de Coatzacoalcos-Jicacal-Las Barrillas & $18^{\circ} 09^{\prime} 30^{\prime \prime}$ & $94^{\circ} 24^{\prime} 57^{\prime \prime}$ \\
\hline
\end{tabular}




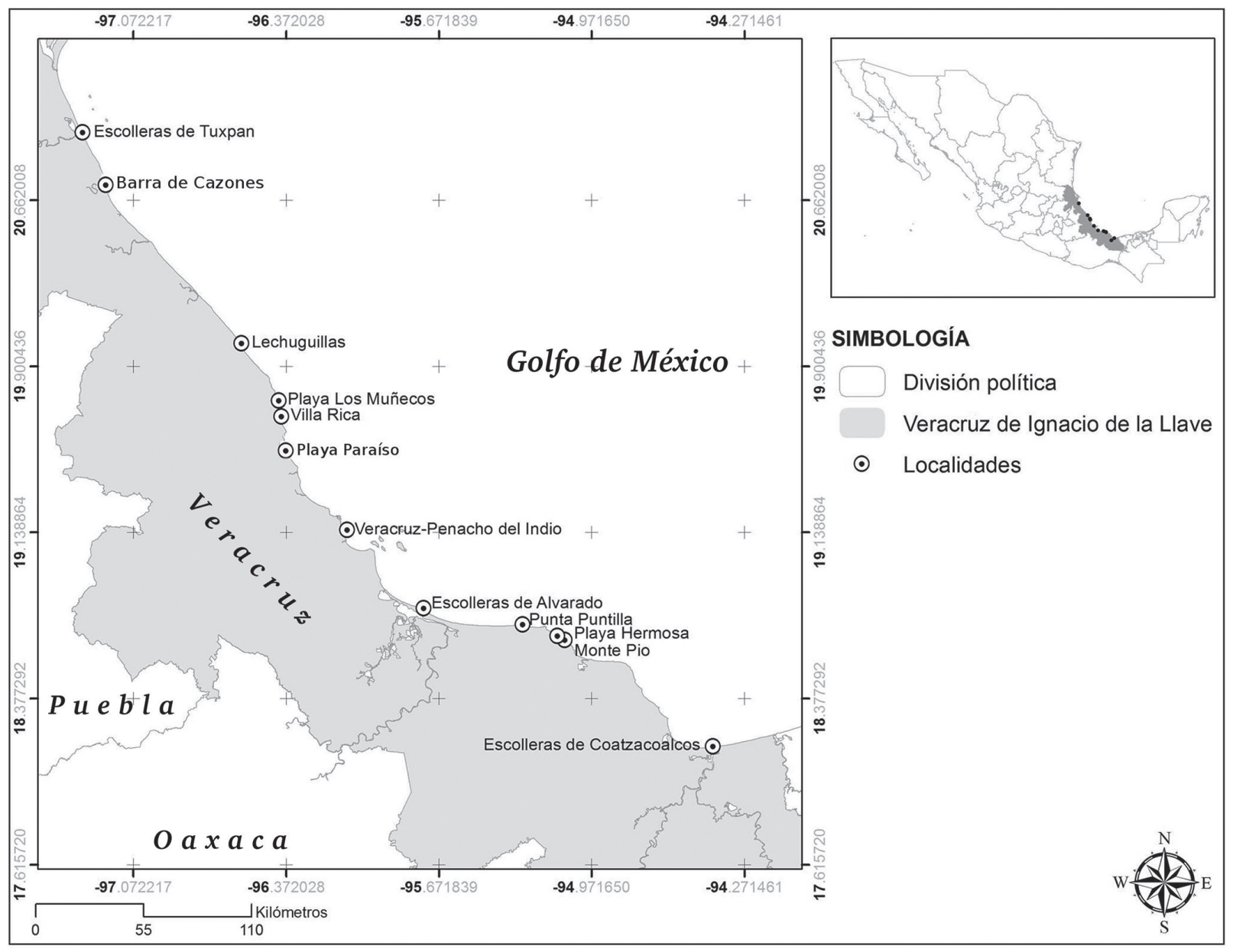

Figura 1. Ubicación del área de estudio y localidades de muestreo. / Location of the study area and sampling localities.

5. Villa Rica: playa con pendiente pronunciada, siendo en su mayoría arenosa con fuerte oleaje y posee un macizo rocoso expuesto.

6. Playa Paraíso: playa arenosa con numerosas formaciones rocosas expuestas a fuerte oleaje.

7. Veracruz-Playa Penacho del Indio: zona principalmente arenosa con agregados rocosos y praderas de pastos marinos.

8. Escolleras de Alvarado: escolleras formadas por grandes rocas, el lugar se encuentra influenciado por actividades de turismo y contaminación por descargas de desechos domésticos.

9. Punta Puntilla: playa preponderantemente arenosa, con agregados rocosos dispersos que en ocasiones forman pozas intermareales, el lugar es una zona de pescadores.

10. Playa Hermosa: playa con pendiente pronunciada, siendo en su mayoría arenosa con fuerte oleaje y posee un macizo rocoso expuesto en donde se forman pozas intermareales.
11. Monte Pío: playa arenosa con agregados rocosos dispersos y restos de formaciones coralinas.

12. Escolleras de Coatzacoalcos-Las Barrillas-Jicacal: escolleras formadas por grandes rocas, además de zonas arenosas protegidas y con agregados de rocas, la localidad se encuentra influenciada por actividades de turismo y presenta contaminación por descargas de desechos domésticos.

TRABAJo DE CAMPO Y GABINETE

Este trabajo incluyó tres fases. La primera fue la recolección de material ficológico en ocho localidades poco estudiadas del litoral de Veracruz, además de Tuxpan, Veracruz-Playa Penacho del Indio, Playa Paraíso y Coatzacoalcos, desde junio de 2006 hasta marzo de 2016, mediante 24 muestreos. Los ejemplares se obtuvieron en diferentes sustratos, tales como rocas, arena y praderas de pastos marinos, principalmente de Thalassia testudinum Banks ex König. 
Las plantas fueron recolectadas a mano con ayuda de espátulas y navajas de campo en el nivel intermareal y solo en Barra de Cazones en el submareal por medio de buceo libre hasta una profundidad de $3 \mathrm{~m}$. Las recolectas se realizaron en segmentos de las playas de aproximadamente $500 \mathrm{~m}$ de extensión.

Las muestras se etiquetaron y se fijaron en una solución de formaldehído a 5\% en agua de mar. El material fue incorporado a la sección ficológica del herbario de la Escuela Nacional de Ciencias Biológicas (ENCB). Para la observación de las algas se utilizó un microscopio binocular Olympus CX31RBSF, realizándose cortes de los talos con navajas de doble filo para distinguir estructuras reproductivas y vegetativas. Para la descalcificación de los representantes de la familia Udoteaceae y Polyphysaceae se utilizó $\mathrm{HNO}_{3}$ 0,6 M. La determinación del material ficológico se llevó a cabo utilizando las claves y descripciones referidas en los trabajos de Taylor (1960), Schneider \& Searles (1991), Littler \& Littler (2000), Dawes \& Mathieson (2008). La secuencia de la lista florística sigue el orden propuesto por Wynne (2011a).

La segunda fase consistió en la revisión del herbario $\mathrm{ENCB}$, donde se obtuvo la información relativa a las especies recolectadas previamente por otros investigadores; también se corrigieron las determinaciones de algunos ejemplares y la tercera fase se refirió a una revisión bibliográfica de los estudios llevados a cabo en la región litoral de Veracruz desde 1962 hasta 2011, con el fin de obtener los registros de algas marinas previamente citados para el litoral del estado y con ello compararlo con los datos obtenidos en el presente estudio. Con la información obtenida se elaboró una lista de especies de algas verdes del litoral de Veracruz, en la que las familias, géneros y especies se ordenaron alfabéticamente. Para las 93 especies ubicadas en este estudio se indican datos de distribución en el área de estudio, estado reproductivo, hábitat, exposición al oleaje, observaciones, referencias y número de herbario ENCB. La actualización nomenclatural se realizó con el esquema propuesto por Wynne (2011a) y Guiry \& Guiry (2016).

\section{RESULTADOS}

FLORÍSTICA

El análisis de las especies de Chlorophyta en el litoral de Veracruz recolectadas y determinadas por las autoras así como el material depositado en el herbario ENCB (82), además de otras 11 especies registradas previamente para el litoral de Veracruz, permitió elaborar una lista florística actualizada que contiene un total de 93 especies, distribuidas en siete órdenes, 20 familias y 28 géneros (Tabla 2). Los géneros con mayor riqueza específica fueron Cladophora (17), Ulva (15) y Caulerpa (12), mientras que los géneros monoespecíficos fueron Phaeophila, Percursaria,
Anadyomene, Boodlea, Phyllodictyon, Dictyosphaeria, Derbesia, Ostreobium, Penicillus, Rhipocephalus, Cymopolia, Gomontia, Ulothrix, Urospora y Stigeoclonium.

En la Tabla 2 se anotan las 82 especies registradas en las 12 localidades de estudio según la clasificación de Wynne (2011a) y Guiry \& Guiry (2016), su distribución en las localidades exploradas, época climática, reproducción, hábitat, referencia y número de colección del herbario ENCB, además de los registros previos para el litoral de Veracruz y que no fueron ubicados por las autoras. Las familias mejor representadas en número de especies fueron Cladophoraceae (28), Ulvaceae (15) y Caulerpaceae (12). Siguen en importancia Bryopsidaceae (7) y Boodleaceae y Codiaceae con 4 especies cada una, Ulvellaceae y Halimedaceae (3), Siphonocladaceae, Udoteaceae, Kornmanniaceae y Polyphysaceae con 2 cada una y finalmente Anadyomenaceae, Dasycladaceae, Ulotrichaceae, Phaeophilaceae y Chaetophoraceae representadas por una especie (Tabla 2).

La integración del listado taxonómico permitió reconocer 93 especies, de las cuales 19 son nuevos registros para el litoral de Veracruz y tres para la costa del Atlántico de México (Tabla 2). Los nuevos registros para Veracruz corresponden a especies de los géneros Phyllodictyon, Cladophora, Urospora, Caulerpa, Chaetomorpha, Penicillus y Trichosolen. Se cita por primera vez a Ulva profunda W.R. Taylor, Chaetomorpha vieillardii (Kützing) M.J. Wynne y Stigeoclonium tenue (C. Agardh) Kützing para la costa Atlántica de México (Tabla 2).

DisTRIBUCIÓN, HÁBITAT Y MODO

La riqueza específica total entre los sitios de muestreo fue diferente: los valores oscilaron desde un máximo de 27 en Barra de Cazones y Playa Penacho del Indio hasta un mínimo de ocho en Playa Paraíso. El mayor número de especies se registró en localidades con áreas rocosas expuestas, como Playa Los Muñecos (24), Punta Puntilla (18) y Coatzacoalcos-Las Barrillas-Jicacal (16). En estos sitios se ubicaron especies de las familias Cladophoraceae, Ulvaceae, Codiaceae, Boodleaceae y Anadyomenaceae. En los sitios con sustrato rocoso y arenoso cubiertos por pastos marinos, de modo protegido, fue donde se encontró la riqueza específica más alta (27 especies). Ejemplo de estas localidades son Barra de Cazones y Playa Penacho del Indio donde se ubicó el número más alto de especies. En estos lugares se encontraron principalmente representantes de las familias Caulerpaceae, Ulvaceae, Bryopsidaceae y Polyphysaceae. En cuanto al establecimiento en sustrato vegetal, se ubicó un total de 19 epífitas que pertenecen a siete familias, siendo Cladophoraceae con siete y Ulvaceae con cinco, las que presentaron el mayor número de representantes epífitos; en conjunto las epifitas representan el $20,4 \%$ de las especies ubicadas en este estudio. 


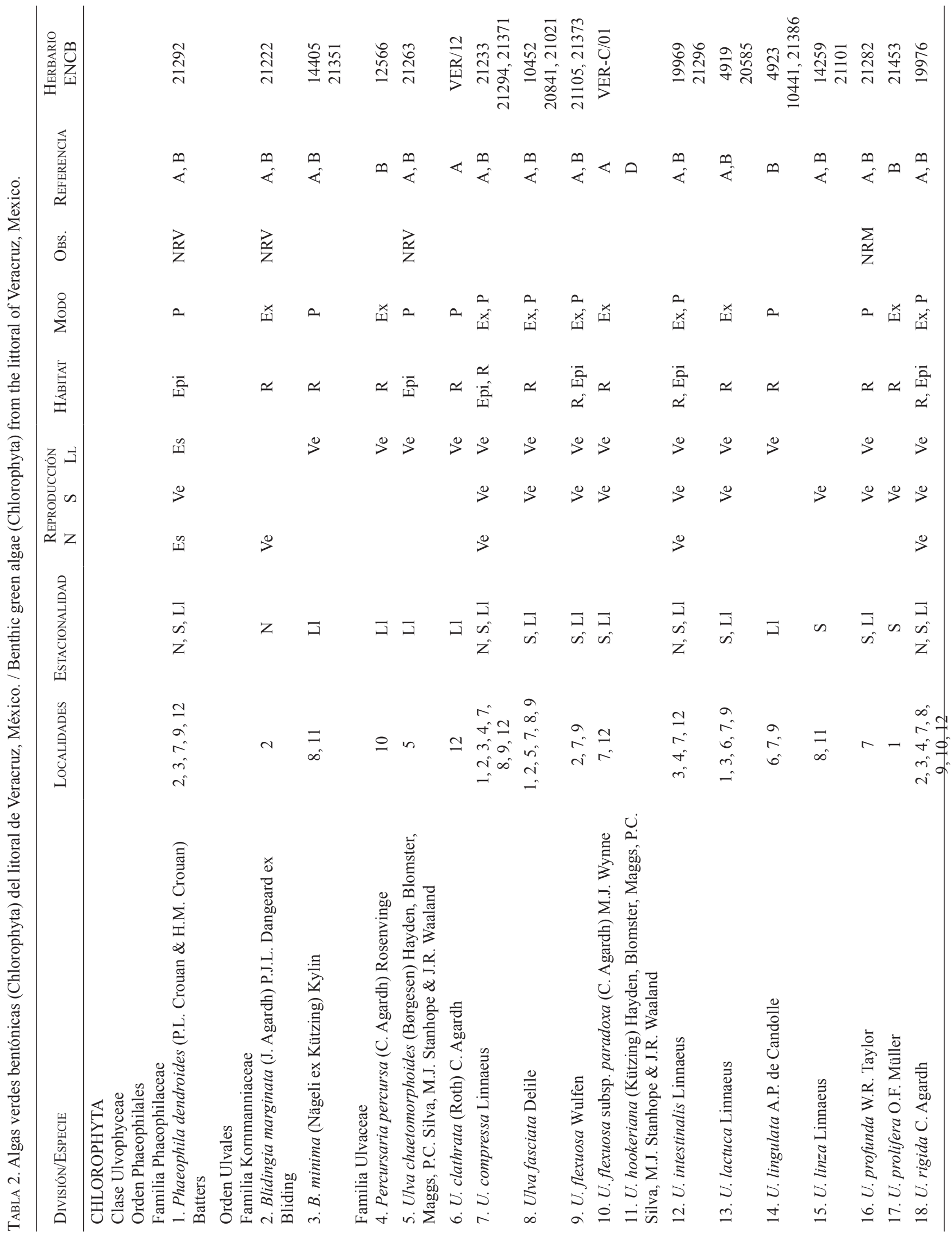




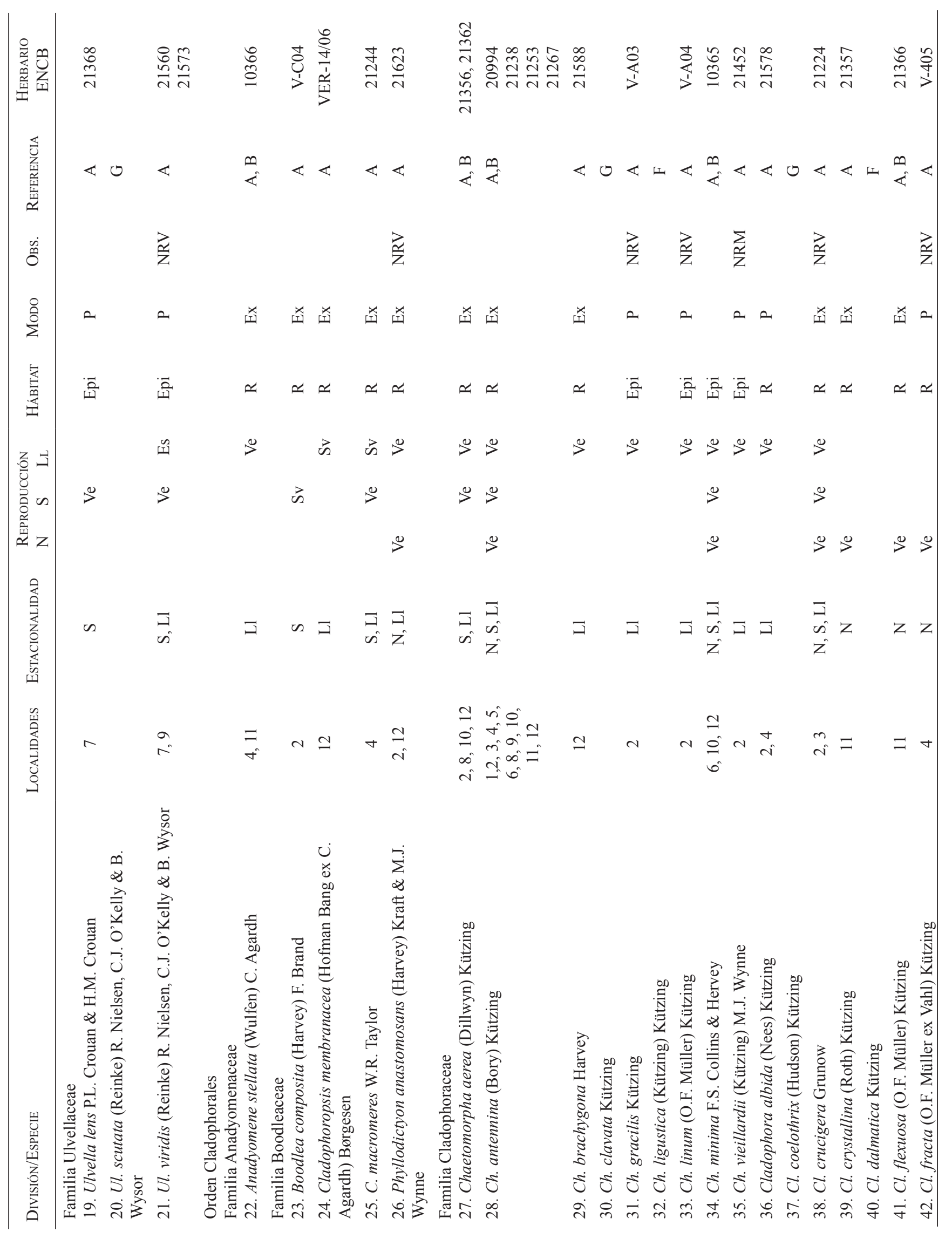




\begin{tabular}{|c|c|c|c|c|c|c|c|c|c|c|c|c|c|c|c|c|c|c|c|c|c|}
\hline 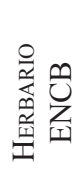 & $\begin{array}{l}\vec{్} \approx \\
\stackrel{2}{\sigma}\end{array}$ & $\begin{array}{l}\stackrel{\infty}{n} \\
\stackrel{\sim}{\sim}\end{array}$ & & $\begin{array}{l}\mathbb{U} \\
\stackrel{\vec{N}}{N}\end{array}$ & $\underset{⿱ n}{\vec{n}}$ & $\begin{array}{l}8 \\
\stackrel{8}{2} \\
\stackrel{2}{N}\end{array}$ & 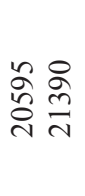 & 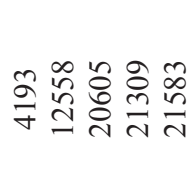 & 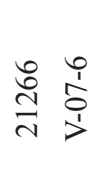 & 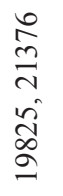 & $\begin{array}{l}\stackrel{\infty}{\circ} \\
\stackrel{2}{2}\end{array}$ & & & $\vec{\sigma}$ & 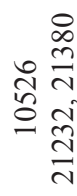 & & $\stackrel{\circ}{\Rightarrow}$ & 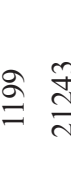 & $\stackrel{\text { du }}{\mathrm{z}}$ & 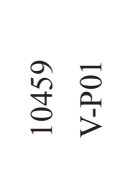 & 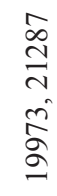 \\
\hline 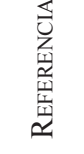 & $\stackrel{n}{<}$ & $\varangle$ & $\ll \infty$ & $\varangle$ & $\begin{array}{l}\infty \\
\ll\end{array}$ & $\varangle$ & $\stackrel{\infty}{<}$ & $\stackrel{m}{<}$ & $\stackrel{m}{\&} \ll$ & $\stackrel{m}{<}$ & $\stackrel{n}{\&}$ & & $\varangle$ & $\stackrel{\infty}{<}$ & $\stackrel{n}{<}$ & $\varangle$ & $\infty$ & $\infty \stackrel{\infty}{\infty}$ & $\infty$ & $\stackrel{n}{\ll} \ll$ & $\stackrel{m}{<}$ \\
\hline$\dot{0}$ & 学 & 学 & 学 & & 总 & & & & & & & & 学 & & & 学 & & & $\frac{\vec{z}}{z}$ & & \\
\hline$\stackrel{\circ}{\stackrel{0}{Z}}$ & $\begin{array}{l}0 \\
x \\
x\end{array}$ & 2 & 盾咸 & $a$ & 爻 & 盾 & 足 & $\begin{array}{l}0 \\
x \\
x\end{array}$ & 2 ․ & 差 & 秘 & & & a & a & 爻 & 爻 & 差夙 & 瓜 & $a$ & $a$ \\
\hline 旨 & $\simeq$ & $\begin{array}{l}\overline{\hat{2}} \\
\text { حي }\end{array}$ & $\simeq \simeq$ & $\simeq$ & $\simeq$ & $\simeq$ & $\simeq$ & $\simeq$ & 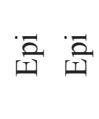 & $\simeq$ & $\simeq$ & & $\simeq$ & $\begin{array}{l}\overrightarrow{\hat{a}} \\
\underline{4}\end{array}$ & $\simeq$ & $\simeq$ & $\simeq$ & $\simeq \simeq$ & $\simeq$ & $\simeq \simeq$ & $\simeq$ \\
\hline ב ב & 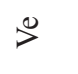 & & $>$ & & 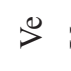 & 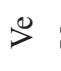 & 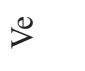 & 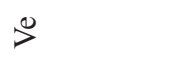 & 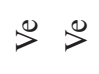 & 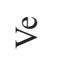 & 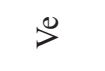 & & & $>$ & $\vec{n}$ & & & $>$ & 8 & हี & ซี \\
\hline 论 & & $\stackrel{1}{10}$ & & & & & 8 & 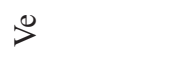 & & & 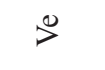 & & & & 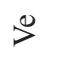 & & 7 & 8 & & & छี \\
\hline$\approx \mathrm{z}$ & & & 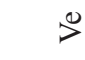 & 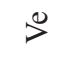 & & & 8 & 8 & & 8 & & & 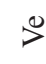 & & ${ }^{\circ}$ & 8 & $>$ & 2 & 8 & & \\
\hline 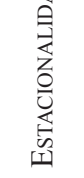 & ב & is & $\exists z$ & Z & $\Xi$ & $\Xi$ & $\begin{array}{l}\vec{z} \\
\dot{n} \\
z\end{array}$ & $\begin{array}{l}\bar{z} \\
\dot{n} \\
z\end{array}$ & コ コ & $\begin{array}{l}\vec{z} \\
z\end{array}$ & $\begin{array}{l}\vec{J} \\
\text { is }\end{array}$ & & $z$ & コ & $\begin{array}{l}\exists \\
\tilde{n} \\
z\end{array}$ & z & z & $\therefore \bar{z}$ & zi & ココ & $\begin{array}{l}\vec{J} \\
\text { is }\end{array}$ \\
\hline $\begin{array}{l}\text { 鱼 } \\
\text { 市 } \\
\text { 荌 } \\
0 \\
0\end{array}$ & $\begin{array}{l}\stackrel{\circ}{\circ} \\
\stackrel{+}{*}\end{array}$ & $\hat{i}$ & $a b$ & N & $N$ & 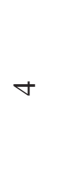 & $\begin{array}{l}\hat{a} \\
\infty \\
\dot{0} \simeq \\
\dot{0} \\
\dot{i} \\
\hat{i}\end{array}$ & 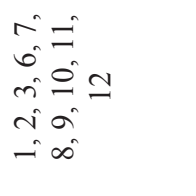 & $\frac{\sim}{a} \infty$ & $\begin{array}{l}\infty \\
i \\
i\end{array}$ & 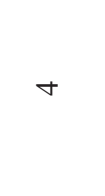 & & $\nabla$ & $\hat{a}$ & $\begin{array}{l}\hat{0} \\
\infty \\
\dot{0}= \\
\hat{n}= \\
\Leftrightarrow\end{array}$ & $\forall$ & 6 & $\begin{array}{r}0 \\
-1 \\
-1\end{array}$ & $\stackrel{0}{a}$ & n & $\hat{\sigma}$ \\
\hline 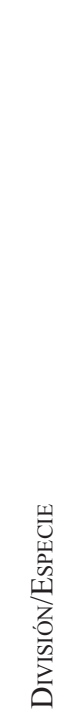 & 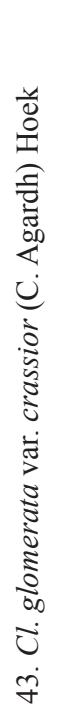 & 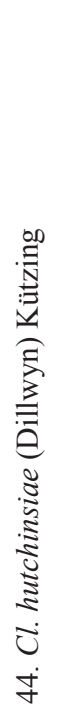 & 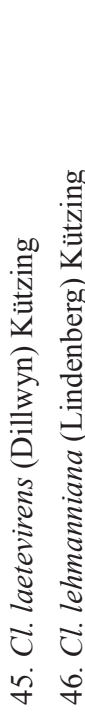 & 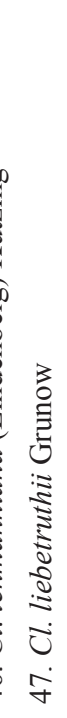 & 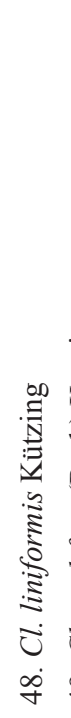 & 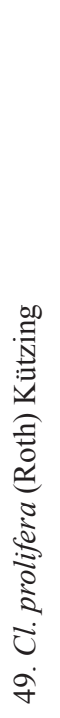 & 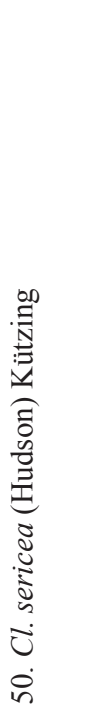 & 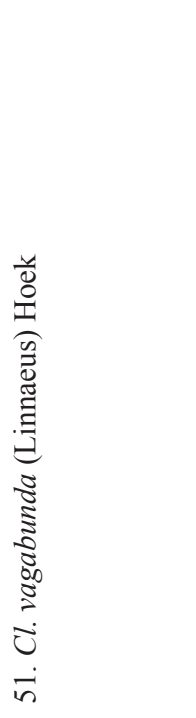 & 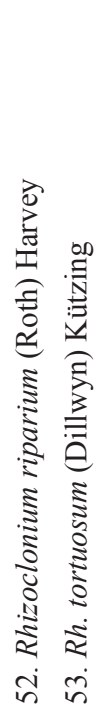 & 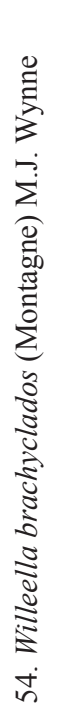 & 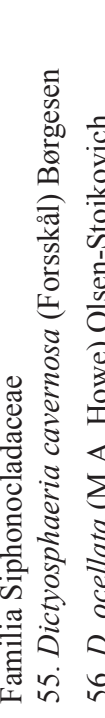 & 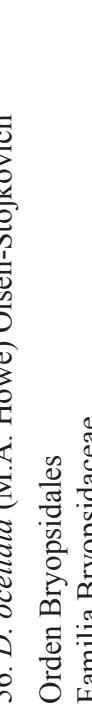 & 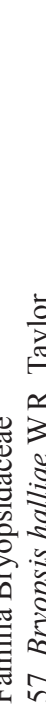 & 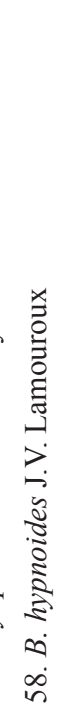 & 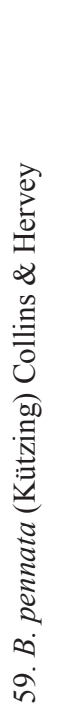 & 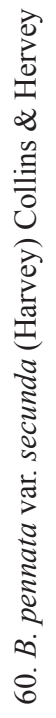 & 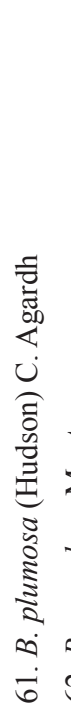 & 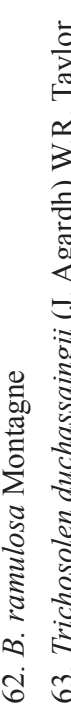 & 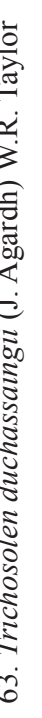 & 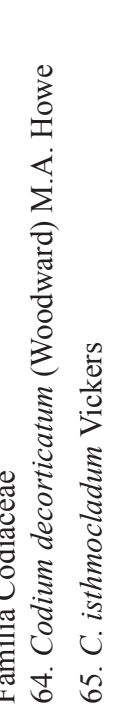 & 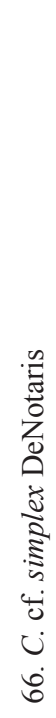 \\
\hline
\end{tabular}




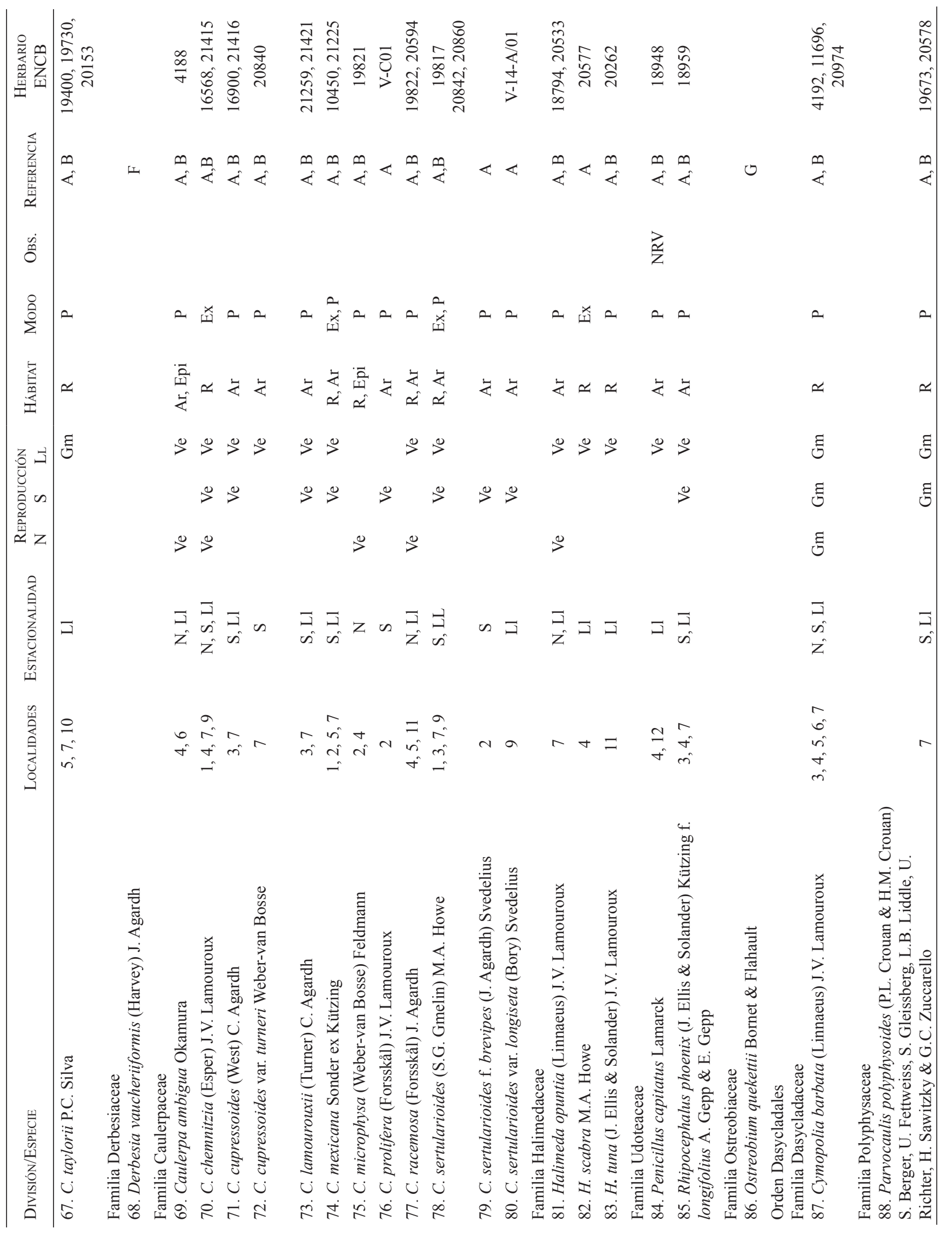


Chlorophyta del Litoral de Veracruz: GARCÍA-LóPEz, D. et al.

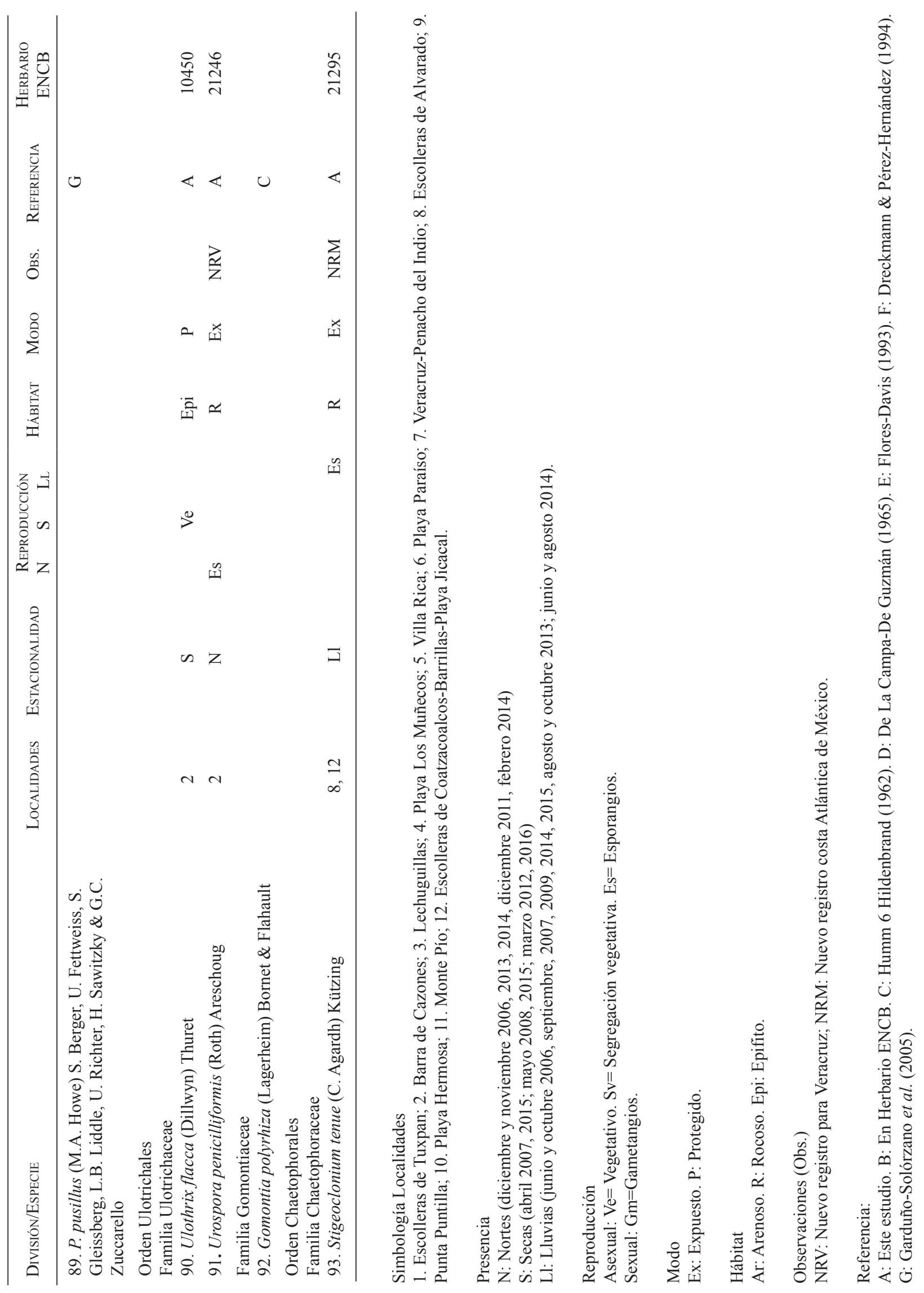




\section{REPRODUCCIÓN}

Del total de taxa registrados en este estudio solo se encontraron a cinco $(5,4 \%)$ en estado reproductivo sexual, presentando gametangios. Los talos fértiles pertenecieron a Codium, Cymopolia, Parvocaulis y Stigeoclonium (Tabla 2). El estado reproductivo asexual lo presentaron ocho especies (8,6 \%): Phaeophila dendroides (P.L. Crouan \& H.M. Crouan) Batters, Ulvella viridis (Reinke) R. Nielsen, C.J. O’Kelly \& B. Wysor, Urospora penicilliformis (Roth) Areschoug y Stigeoclonium tenue (C. Agardh) Kützing (Figs. 4a, b), se encontraron con esporangios; mientras que Boodlea composita (Harvey) F. Brand, Cladophoropsis membranacea (Hofman Bang ex C. Agardh) Børgesen, C. macromeres W.R. Taylor y Bryopsis pennata (Kützing) Collins \& Hervey presentaron segregación vegetativa. El resto de las especies $(86,0 \%)$ se halló en estado vegetativo.

VARIACIÓN POR ÉPOCA CLIMÁTICA

Del total de las algas determinadas, 58 se registraron en la época climática de lluvias de verano (incremento correspondiente a los representantes del Orden Cladophorales), seguido de la época de secas con $37 \mathrm{y}$ lluvias de invierno (Nortes) con 28 especies.

\section{DISCUSIÓN}

FLORÍSTICA

Las Chlorophyta determinadas en este trabajo, a excepción de Urospora penicilliformis y Stigeoclonium tenue, se encuentran distribuidas en el Atlántico Tropical Occidental, desde el sur de Florida hasta Cabo Frio, Brasil (Taylor 1960, Littler \& Littler 2000, Ortega et al. 2001, Wynne 2011a). El número total de especies encontradas en este estudio (93) es mayor que el registrado por Mateo-Cid et al. (2013) para el litoral de Campeche, quienes registraron 76 especies de Chlorophyta en esa región.

Los resultados obtenidos sugieren que el litoral de Veracruz es un área con relativa alta diversidad florística, si se tiene en cuenta que en su línea de costa se puede encontrar el 51,6 \% de las Chlorophyta conocidas para la costa Atlántica de México que es de 180 (Garduño-Solórzano et al. 2005, Mateo-Cid et al. 2013, Acosta-Calderón et al. 2016). Es importante resaltar que la variedad de microambientes, combinada con características oceanográficas particulares, actúan en conjunto en el área de estudio y proveen las condiciones para el desarrollo de un elevado número de algas verdes (Pedroche \& Sentíes 2003, Cetz-Navarro et al. 2008).

Once especies previamente registradas para el litoral de Veracruz no fueron ubicadas en el presente trabajo. Esta situación puede deberse a varios factores: 1) a la aplicación de nombres incorrectos derivado de la falta de certeza de los caracteres taxonómicos actualmente en uso para la determinación de las especies (Ortega et al. 2001), específicamente Fama et al. (2002) y Espinoza-Avalos et al. (2015) mencionan que existe un complejo de especies del género Caulerpa que requiere de revisión taxonómica; 2) algunas algas presentan ciclos de vida heteromorficos, como Derbesia vaucheriformis (Lee 2008), en el que la fase macroscópica tal vez no se encuentre durante los meses de muestreo; 3) período de recolecta, tipo de muestreo y localidades exploradas; 4) eventos climatológicos como huracanes, los cuales pueden inducir cambios en los hábitats marinos, y juegan un rol importante en el clima del mundo, al transportar el exceso de calor y humedad de los trópicos, a latitudes templadas y boreales, además alteran el clima local a mediano plazo (Lugo 2000).

Nuevos Registros

De los 72 taxa registrados previamente para el litoral de Veracruz (Garduño-Solórzano et al. 2005) se ubicaron a 61; 19 son nuevos registros para Veracruz y tres para la costa Atlántica de México: 1) Ulva profunda (Figs. 2c, d, e) ha sido registrada en Cuba y Florida y tiende a ser confundida con Ulva rigida; 2) El nombre de Chaetomorpha crassa (C. Agardh) Kützing ha sido aplicado a especímenes flotantes o epifitos con filamentos gruesos que habitan en los mares tropicales y templados de América; de acuerdo con Wynne (2011b), estos especímenes corresponden a Chaetomorpha vieillardii (Figs. 3c, d), especie que se distribuye en Brasil, Filipinas y Nueva Caledonia, mientras que en México no ha sido registrada debido a que probablemente es confundida con Ch. crassa; 3 ) en el caso de Stigeoclonium tenue (Figs. 4a, b), Francke \& Rhebergen (1982) en su trabajo sobre ecotipos eurihalinos de algunas especies de Stigeoclonium de Schiermonnikoog, una de las islas Frisias occidentales, en los Países Bajos, encontraron poblaciones de Stigeoclonium tenue en ambientes salobres; además individuos de $S$. tenue se aislaron y en laboratorio fueron expuestos a diferentes salinidades. Estos autores determinaron que $S$. tenue presenta tolerancia a intervalos de salinidad entre 1 y 18 u.p.s., lo que se atribuye a la plasticidad fisiológica de algunas especies de algas, hecho que se ha demostrado en Rhizoclonium riparium y Bangia atropurpurea (Mertens ex Roth) C. Agardh, especies capaces de tolerar un amplio intervalo de salinidad, pudiendo ser ubicadas tanto en ambientes salobres como marinos. Por otro lado, los autores también observaron cambios morfológicos de los talos de Stigeoclonium tenue sometidos a diferentes salinidades, tales como la reducción general del sistema erecto y la disociación de los filamentos postrados y erectos en grupos de partículas esféricas. Por lo anterior, es posible deducir que la influencia de descargas de aguas domésticas en escolleras y el aporte de agua dulce propio de la época de lluvias probablemente influyó en la presencia de Stigeoclonium tenue en Coatzacoalcos y Alvarado. 

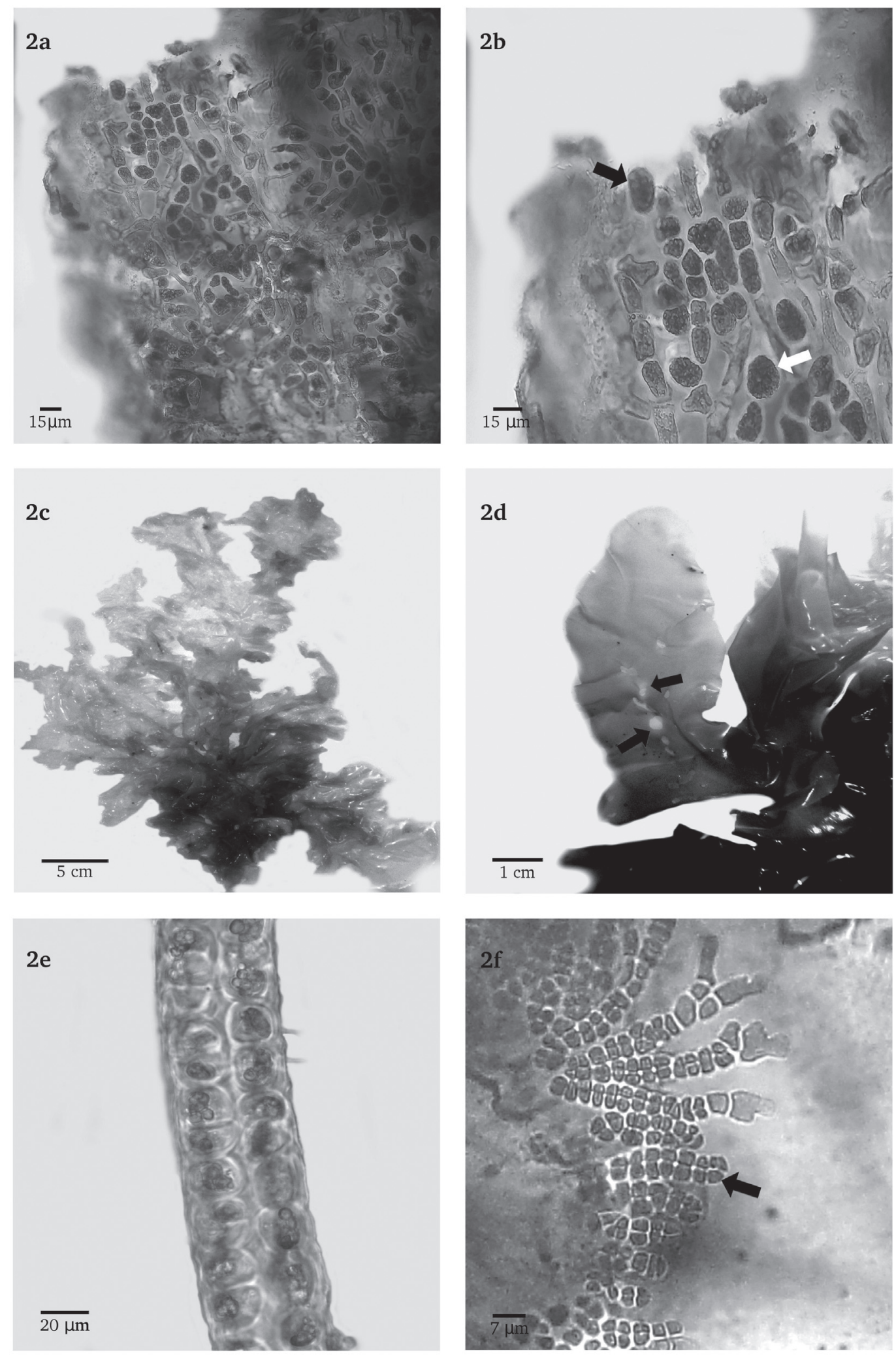

Figura 2. 2a: Phaeophila dendroides aspecto general del talo; 2b: Detalle de los esporocistos (flechas); 2c: Ulva profunda, aspecto general; 2d: Perforaciones de la lámina (flecha); 2e. Corte transversal de la lámina; $2 \mathrm{f}$ : Ulvella viridis detalle de los filamentos postrados con esporocistos (flecha). / 2a: Habit of Phaeophila dendroides; 2b: Detail of the sporocysts (arrows); 2c: Habit of Ulva profunda; 2d: Blade perforations (arrow); 2e. Transversal section of blade; 2f. Ulvella viridis detail of the filaments prostrate with sporocysts (arrow). 

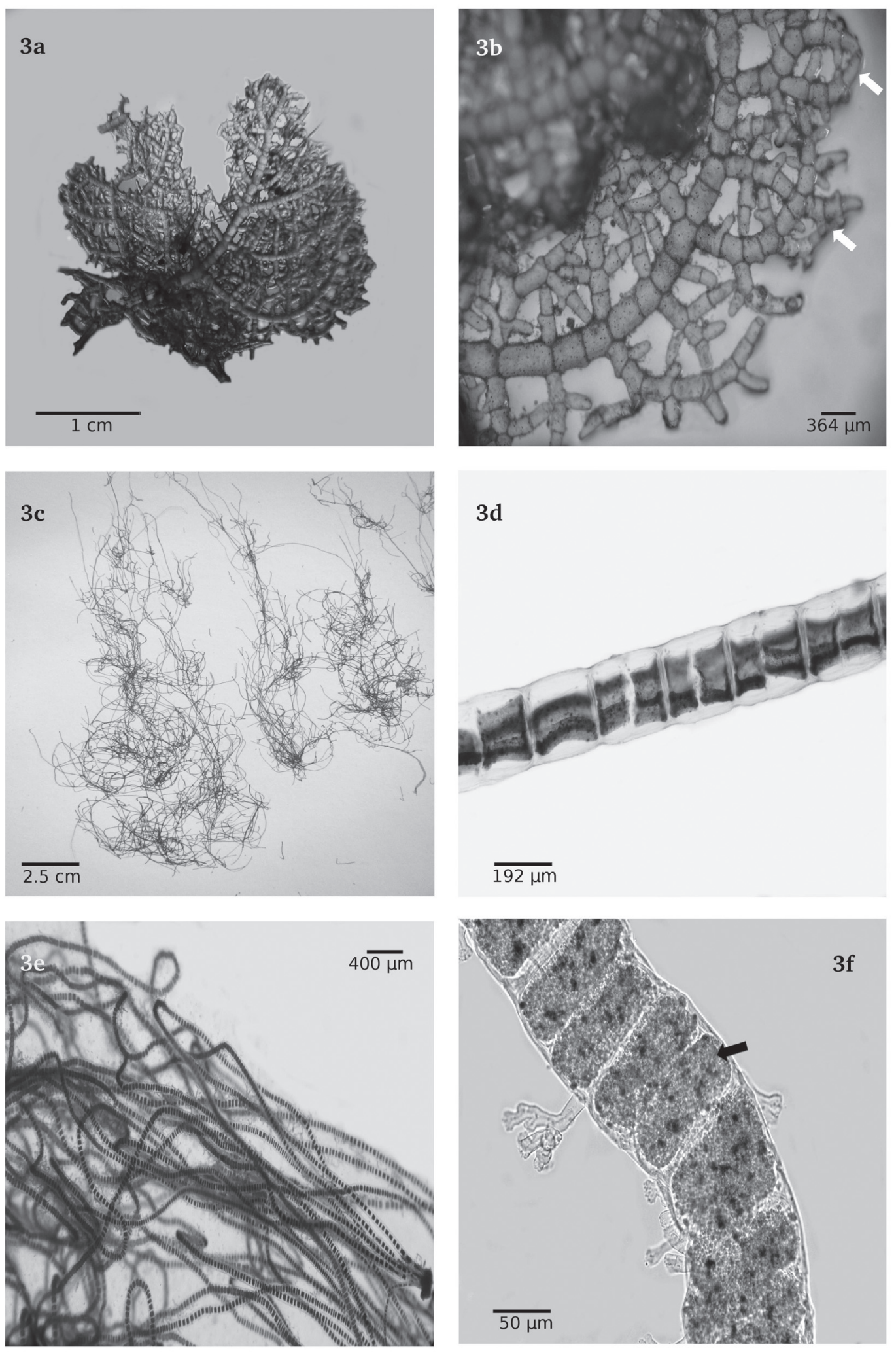

Figura 3. 3a: Phyllodictyon anastomosans, aspecto general del talo; 3b: Detalle de la porción apical del talo (flechas); 3c: Chaetomorpha vieillardii, aspecto general del talo, 3d: Detalle de los filamentos uniseriados; 3e: Urospora penicilliformis, aspecto general; $3 \mathrm{f}$ : Detalle del cloroplasto (flecha). / 3a: Habit of Phyllodictyon anastomosans; 3b: detail of apical portions (arrows); 3c: Habit of Chaetomorpha vieillardii; 3d: Detail of uniseriate filament; 3e: Habit of Urospora penicilliformis; 3f: Detail of chloroplast (arrow). 

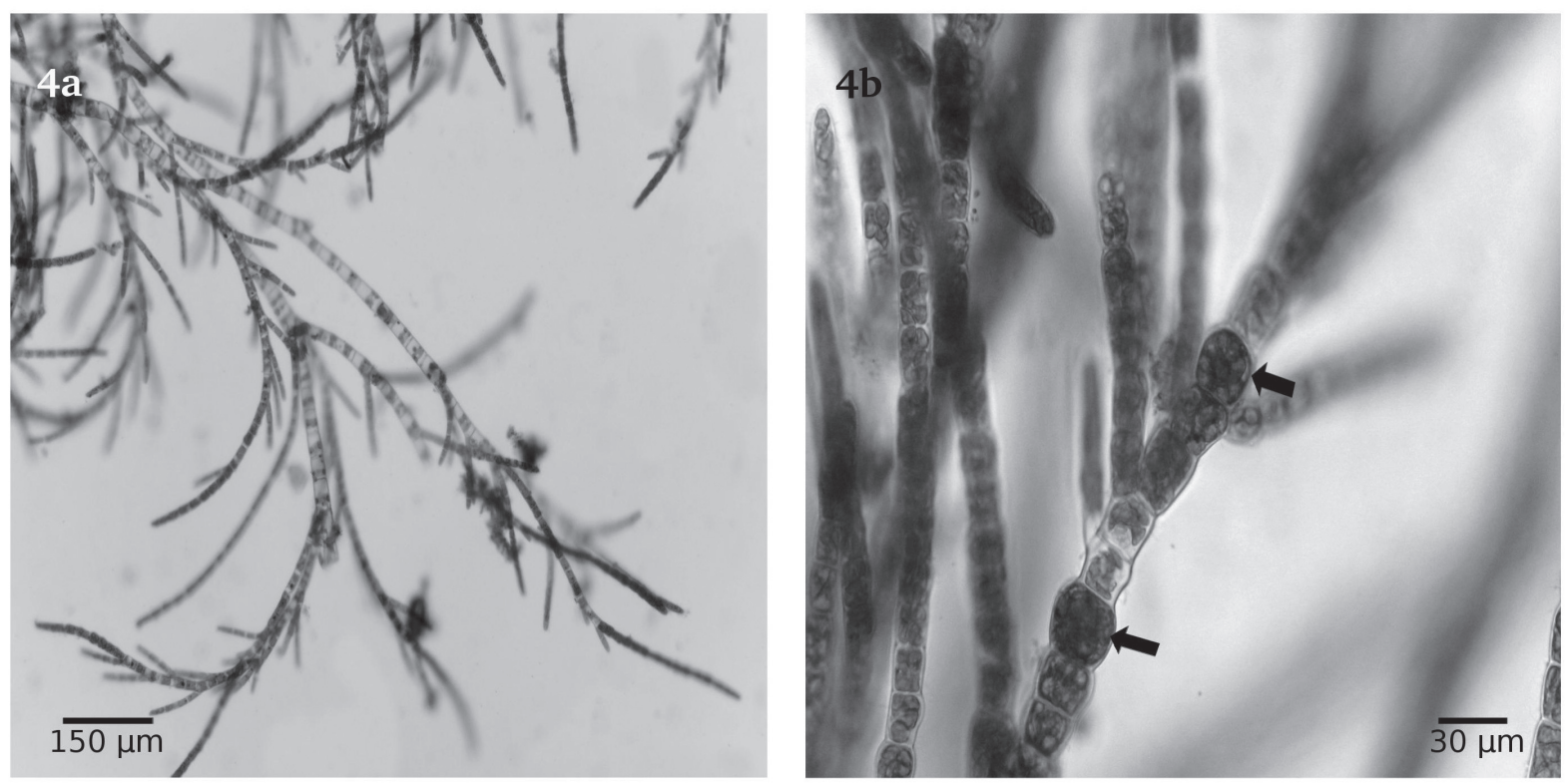

Figura 4. 4a: Stigeoclonium tenue aspecto general; 4b: Detalle de los filamentos y esporocistos (flechas). / 4a: Habit of Stigeoclonium tenue; 4b: Detail of the filaments with sporocysts (arrows).

Entre los nuevos registros para el litoral de Veracruz destacan Phaeophila dendroides (Figs. 2a, b) y Ulvella viridis (Fig. 2f), ambas de hábito epífito, Phyllodictyon anastomosans (Figs. 3a, b), especie con escasos registros en el Atlántico mexicano, mientras que dentro del género Cladophora se encontro el mayor número de nuevos registros con seis, sobresaliendo $\mathrm{Cl}$. crucigera, Cl. fracta, $\mathrm{Cl}$. hutchinsiae y $\mathrm{Cl}$. liniformis.

En suma, el listado florístico de Chlorophyta para el litoral de Veracruz está constituido por 93 taxa. El alto número de nuevos registros encontrados en el área de estudio puede deberse a: 1) Las localidades de muestreo que no habían sido previamente estudiadas, 2) La revisión minuciosa del material recolectado y del material de herbario, lo que permitió ubicar a especies epífitas, y 3) El número (24) de recolectas llevadas a cabo.

\section{DISTRIBUCIÓN, HÁBITAT Y MODO}

Las diferencias en la riqueza específica entre las localidades de muestreo pueden ser explicadas por el tipo de sustrato dominante en cada uno de los sitios; por ejemplo, en la Playa Los Muñecos y Coatzacoalcos-Barrillas-Jicacal, lugares con sustrato rocoso, se registraron 24 y 16 especies respectivamente, lo que concuerda con Garduño-Solórzano et al. (2005), quienes encontraron que las algas verdes que habitan en las costas mexicanas del Golfo de México y Mar Caribe se desarrollan principalmente sobre sustratos duros; Santelices (1977) indica que los sustratos duros y estables permiten la fijación y desarrollo de un mayor número de algas marinas bentónicas.

De manera similar, las localidades protegidas con fondos rocosos y arenosos asociados a Thalassia testudinum presentaron un mayor número de especies, como VeracruzPlaya Penacho del Indio, Barra de Cazones, ambas con 27, mientras que en Lechuguillas y Punta Puntilla se registraron 14 y 18 especies, respectivamente, siendo las dos primeras los sitios de muestreo con la riqueza específica más alta del litoral de Veracruz. En estos lugares se ubicaron a especies del género Caulerpa, que presentan adaptaciones que les permiten vivir en este tipo de hábitats como el desarrollo de rizoides o mazas rizoidales que penetran en el sustrato blando y la producción de metabolitos secundarios para evitar la herbivoría (Lobban \& Harrison 1997). En el caso de las escolleras de Tuxpan y Alvarado, localidades expuestas a fuerte oleaje, se determinaron 13 y 12 especies de Chlorophyta, respectivamente; este tipo de sustrato es considerado poco adecuado para el desarrollo de las algas por la inestabilidad ante una fuerza mecánica como el oleaje, corrientes y mareas que puede llegar a mover el sustrato, incrementando la turbidez o enterrando a las algas, además de que estas localidades se encuentran en zonas donde existen aportes de agua dulce que disminuyen la salinidad hasta valores de ambientes salobres (Santelices 1977, Garduño-Solórzano et al. 2005).

Con respecto a Villa Rica y Monte Pío, se encontraron 9 especies y en Playa Hermosa y Playa Paraíso, la riqueza 
específica fue de 8 y 9 especies, respectivamente, lo cual puede atribuirse a que las playas son mayormente arenosas sin cobertura de T. testudinum, y con intenso oleaje.

\section{ESPECIES EPIFITAS}

Se ha observado que el establecimiento de algas epífitas sobre los diferentes hospederos depende tanto de factores climáticos estacionales como de aspectos biológicos, como la superficie del hospedero o la producción de sustancias extracelulares como estrategia de defensa ante la herbivoría, que las vuelven poco atractivas para las epífitas (Round 1981). En este estudio se encontraron 19 especies de hábito epífito, especialmente en algas rojas como Digenea simplex (Wulfen) C. Agardh (Mateo-Cid et al. 2013), la cual fue encontrada como hospedero de especies de los géneros Ulva, Ulvella, Phaeophila, Chaetomorpha y Cladophora (Tabla 2). Santelices (1977) ha sugerido que la longevidad del hospedero debe ser lo suficientemente larga para permitir a las epífitas completar su ciclo de vida.

La importancia biológica de las algas epífitas reside en que participan en la cadena y redes tróficas de diversos organismos marinos que habitan en los litorales intermareales (Ramírez-Rodríguez et al. 2011) y en que la interacción epífita-hospedero presenta efectos negativos como la reducción de energía lumínica, y por tanto de la actividad fotosintética del hospedero y positivos, como la protección de los talos por parte de las epífitas (Franschetti et al. 2002).

\section{REPRODUCCIÓN}

En este estudio se ubicó un bajo número de especies de Chlorophyta en estado reproductivo, hecho que coincide con lo reportado en diversos estudios florísticos realizados en las costas mexicanas (Mateo-Cid et al. 2013, AcostaCalderón et al. 2016). Esta situación puede deberse a que los mecanismos de reproducción de este grupo de algas implican la rápida formación de estructuras de reproducción y liberación de gametos móviles (Acosta-Calderón et al. 2016), lo cual solo se podría registrar si los días de reproducción sexual coincidieran con el tiempo de recolecta. Así, Coppejans et al. (2001) mencionaron que la aparición de estructuras de reproducción en Ulvophyceae es estacional, mientras que Clifton (1997) observó que los eventos de reproducción sexual de las Bryopsidales en arrecifes coralinos implica un rápido desarrollo de gametangios y liberación de gametos durante las primeras horas del día o durante la noche. Las algas en estado fértil que se ubicaron en este trabajo pertenecen a los géneros Phaeophila, Ulvella, Codium, Cymopolia, Parvocaulis, Urospora, Stigeoclonium, Boodlea, Bryopsis y Cladophoropsis.

VARIACION POR ÉPOCA CLIMÁTICA

La mayor riqueza específica se presentó en lluvias de verano (58), lo cual puede estar relacionado con la disponibilidad de luz. Lalli \& Parson (1997) mencionan que en latitudes tropicales la superficie del agua de mar recibe más energía del sol durante verano, en comparación a invierno y los días de verano son cuatro o cinco horas más largos, lo que se traduce en una mayor energía disponible para la fotosíntesis de las algas. En contraparte, el menor número de especies se registró en la temporada de nortes (28); durante este periodo la energía proveniente del sol es menor (Lalli \& Parson 1997). Suárez (1989) plantea que los cambios estacionales en la temperatura del agua de mar en la plataforma cubana inciden en la riqueza de especies, con una disminución en los meses fríos (octubre-noviembre) y un aumento en los meses más cálidos (junio-agosto). El efecto de la luz y la temperatura sobre la variaciones temporales de la composición y riqueza de las algas marinas bentónicas está ampliamente documentado (Santelices 1977), otros factores como la precipitación, nutrientes, cambios en la dinámica del agua producto de los frentes fríos y tormentas, también inciden en el desarrollo de la flora marina (Santelices 1977, Suárez 1989).

\section{CONCLUSIONES}

La realización de estudios como el presente, además de mostrar la riqueza específica de las algas verdes en el litoral veracruzano resalta la relevancia de realizar investigaciones periódicas en esta región, ya que se ubicaron un alto número de nuevos registros tanto en las ocho localidades poco estudiadas como en las que ya se habían realizado estudios.

\section{RECOMENDACIONES}

El análisis y la integración de los estudios previos y los resultados obtenidos en el presente estudio permiten destacar la importancia de realizar inventarios ficoflorísticos en regiones del litoral mexicano poco investigadas. Además, se recomienda continuar con los muestreos en las localidades visitadas para recabar datos de los cambios espaciales y temporales de la flora, que permitan monitorear e identificar posibles alteraciones en las condiciones ambientales del litoral veracruzano, ya sea por condiciones climáticas naturales tales como huracanes o períodos de lluvias intensos, así como por las actividades de turismo, pesquerías y aumento de los efectos causados por el incremento de la población humana en la zona costera de Veracruz.

\section{AGRADECIMIENTOS}

Al Instituto Politécnico Nacional (SIP-20161042, 20164772, 20170696 y 20170767), por facilitar el apoyo económico, las instalaciones y el equipo necesario para el desarrollo 
de este estudio. La segunda y tercera autora agradecen la beca otorgada por la Comisión de Operación y Fomento de Actividades Académicas (COFAA). LEMC y ACMG reconocen los incentivos del programa EDI-IPN. A Rocío Nava Olvera y Julio A. Acosta Calderón por su apoyo en el trabajo de campo, a Xhail Flores Leyva por la realización de la figura del área de estudio.

\section{REFERENCIAS}

Acosta-Calderon, J.A., Mateo-Cid, L.E., Mendoza-Gonzalez, A.C. 2016. An updated list of marine green algae (Chlorophyta, Ulvophyceae) from the Biosphere Reserve of Sian Ka'an, Quintana Roo, Mexico. Check List 12 (3): 1886. DOI: http://dx.doi.org/10.15560/12.3.1886

Carranza-Edwards, A., Gutiérrez-Estrada, M., RodríguezTORRES, R. 1975. Unidades morfotectónicas continentales de las costas mexicanas. Anales del Centro de Ciencias Marinas y Limnología 2(1): 81-88.

Cetz-Navarro, N., Espinoza-Ávalos, J., Sentíes-Granados, A., QuAN-YounG, L. 2008. Nuevos registros de macroalgas para el Atlántico Mexicano y riqueza florística del Caribe Mexicano. Hidrobiológica 18: 11-19.

Clifton, K. 1997. Mass spawning by green algae on coral reefs. Science 275: 1116-1118.

Coppejans, E., Leliaert, F., Dargent, O., Clerck, O. 2001. Marine green algae (Chlorophyta) from the north coast of Papua New Guinea. Cryptogamie 22: 1-69.

Dawes, C.J., Mathieson, A.C. 2008. The seaweeds of Florida. University Press of Florida. Gainesville, USA. 591 pp.

De la Campa-De Guzmán, S. 1965. Notas preliminares sobre un reconocimiento de la flora marina del estado de Veracruz. Anales del Instituto Nacional de Investigaciones Biológicas Pesqueras 1: 9-49.

De la Lanza-Espino, G., Gómez-Rojas, J.C. 2004. Características físicas y químicas del Golfo de México. En: Caso, M., Pisanty, I., Ezcurra, E. (eds.), Diagnóstico ambiental del Golfo de México. Vol. 1, pp. 105-136. Secretaría del Medio Ambiente y Recursos Naturales, Instituto Nacional de Ecología, AC. - Harte Research Institute for Gulf of Mexico Studies. D.F. México, México.

Dreckmann, K.M., Pérez-Hernández, M.A. 1994. Macroalgas bentónicas de la laguna de Tampamachoco, Veracruz, México. Revista de Biología Tropical 42: 715-717.

Espinoza-Avalos, J., Aguilar-Rosas, L.E., Aguilar-Rosas, R., Gómez-Poot, J.M., Raigoza-Figueras, R. 2015. Presencia de Caulerpaceae (Chlorophyta) en la península de Yucatán, México. Botanical Sciences 93(4): 845-854.

Fama, P., Wysor, B., Kooistra, W., Zucarello, G. 2002. Molecular phylogeny of the genus Caulerpa (Caulerpales, Chlorophyta) inferred from chloroplast tufA gene. Journal of Phycology 38: 1040-1050.

Flores-Davis, J.G. 1993. Clorofíceas del Litoral rocoso de La Mancha, Veracruz. Secretaría de Educación y Cultura, Dirección General de Educación Media Superior y Superior, Xalapa. 94 pp.

Francke, J.A., Rhebergen, L.J. 1982. Euryhaline ecotypes in some species of Stigeoclonium Kütz. British Phycological
Journal 17: 135-145.

Franschetti, S., Giangrande, A., Terlizzi, A., Miglietta, M.P., Della Tommassa, L., Boero, F. 2002. Spatio-temporal variation of hydroids and polychaetes associated with Cystoseira amentacea (Fucales: Phaeophyceae). Marine Biology 140: 949-957.

GARcía, E. 1983. Modificaciones al sistema de clasificación climática de Köppen (para adaptarlo a las condiciones de la República Mexicana). Instituto de Geografía, Universidad Nacional Autónoma de México. México, D.F., México. 246 pp.

Garduño-Solórzano, G., Godínez, J.L., Ortega, M.M. 2005. Distribución geográfica y afinidad por el sustrato de las algas verdes (Chlorophyceae) bénticas de las costas mexicanas del Golfo de México y Mar Caribe. Boletín de la Sociedad Botánica de México 76: 61-78.

Guiry, M.D., Guiry, G.M. 2016. Algaebase version 4.2. Worldwide electronic Publication. National University of Ireland. Galway, Ireland. http://www.algaebase.org.

Huerta-Múzquiz, L. 1960. Lista preliminar de las algas marinas del litoral del Estado de Veracruz. Boletín de la Sociedad Botánica de México 25: 39-45.

Humm, H.J., Hildebrand, H.H. 1962. Marine algae from the Gulf Coast of Texas and Mexico. Publications of the Institute of Marine Science 8: 227-268.

Lalli, C., Parson, T. 1997. Biological Oceanography. An Introduction. Open University. Butterworth Heinemann. $320 \mathrm{pp}$.

LeE, R. 2008. Phycology. Cambridge University Press, New York. $550 \mathrm{pp}$.

Liebmann, F.M. 1846. Beretning om Amerikas Vandplanter af Algernes Classe. Oversigt over det Kongelige Danske Videnskabernes Selskabs Forhandlinger og dets Meldemmers Arbeider 1846: 72-77.

Littler, D.S., Littler, M.M. 2000. Caribbean reef plants. An identification guide to the reef plants of the Caribbean, Bahamas, Florida and Gulf of Mexico. Offshore Graphics, Inc., Washington D.C. 542 pp.

Lobban, C., Harrison, P. 1997. Seaweed ecology and physiology. Cambridge University Press, New York. 366 pp.

Lugo, A. 2000. Effects and outcomes of Caribbean hurricanes in a climate change scenario. Science of the Total Environment 262: 243-251.

Mateo-Cid, L.E., Mendoza-GonzÁlez, A.C., Ávila-Ortiz, A.G., Díaz Martínez, S. 2013. Algas marinas bentónicas del litoral de Campeche, México. Acta Botanica Mexicana 104: 53-92.

Ortega, M.M., Godínez, J.L., Garduño-Solórzano, G. 2001. Catálogo de algas bénticas de las costas del Golfo de México y mar Caribe. Universidad Nacional Autónoma de México. México, D.F., México. 594 pp.

Pedroche, F., Sentíes, A. 2003. Ficología marina mexicana. Diversidad y problemática actual. Hidrobiológica 13: 2332.

Ramírez-Rodríguez, M.L.A. 1975. Contribución al conocimiento de las algas marinas del litoral rocoso de Villa Rica, Veracruz, tesis profesional, Facultad de Biología, Universidad Veracruzana, Xalapa. $65 \mathrm{pp}$.

Ramírez-Rodríguez, M.L.A., Blanco Pérez, R., Okolodkov, Y.D. 2011. Diversidad de especies de algas epífitas 
marinas. 2011. En: La Biodiversidad en Veracruz: Estudio de Estado, vol. 2. CONABIO, Gobierno del Estado de Veracruz, Universidad Veracruzana, Instituto de Ecología, pp.71-76, Apéndice VIII.3. Lista de especies de algas epífitas en los litorales de Veracruz. $11 \mathrm{pp}$.

Round, F.E. 1981. The ecology of the algae. Cambridge University Press, Nueva York. 653 pp.

SÁnchez-Rodríguez, M.E. 1967. Flora marina de Monte Pío, Estado de Veracruz, México, Anales de la Escuela Nacional de Ciencias Biológicas, México 14(14): 9-18.

SÁncheZ-RodríguEZ, M.E. 1980. Ficoflora del sustrato rocoso dentro de las costas del Golfo de México, México. Boletim do Instituto Oceanográfico (São Paulo) 29:347-350.

SAntelices, B. 1977. Ecología de algas marinas bentónicas. Efectos de factores ambientales. Pontificia Universidad Católica de Chile. Chile. 303 pp.

Schneider, C.W., Searles, R.B. 1991. Seaweeds of the southeastern United States. Cape Hatteras to Cape Cañaveral. Duke
University Press. Durham, USA. 563 pp.

SuÁrez, A.M. 1989. Ecología del macrofitobentos de la plataforma de Cuba. Revista de Investigaciones Marinas 10: 187-203.

TAYLOR, W.R. 1960. Marine algae of the eastern tropical and subtropical coasts of the Americas. University of Michigan, Ann Arbor, USA. xi +870 pp.

ValenZuela, D.H. 1987. Contribución al conocimiento de la vegetación marina del litoral rocoso de Playa Escondida, Veracruz. Tesis de licenciatura. Instituto Politécnico Nacional, Escuela Nacional de Ciencias Biológicas. México, D.F., Mexico. 112 pp.

Wynne, M.J. 2011a. A checklist of benthic marine algae of the tropical and subtropical Western Atlantic: third revision. Nova Hedwigia Beih. 140: 1-166.

Wynne, M.J. 2011b. Proposal of the name Chaetomorpha vieillardii (Kütz.), n. comb., for a large-celled tropical Chaetomorpha (Chlorophyta). Pacific Science 65(1): 109-115.

Recibido: 14.10.2016

Aceptado: 13.03.2017 